\title{
Consecuencias del derrame pleural
}

\author{
E. MONTERO RUIZ, C. HERNÁNDEZ AHIJADO, F. GARCÍA SÁNCHEZ, \\ J. LÓPEZ ÁLVAREZ \\ Servicio de Medicina Interna. Hospital Universitario Príncipe de Asturias. Alcalá de \\ Henares. Madrid
}

\author{
EFFECTS OF PLEURAL EFFUSION
}

\begin{abstract}
RESUMEN
El derrame pleural (DP) modifica el equilibrio entre el volumen de la cavidad torácica y el de sus órganos, alterando el funcionamiento del aparato respiratorio, corazón y diafragma. Altera poco el intercambio pulmonar de gases, pero provoca cambios restrictivos proporcionales a su volumen, aumenta los diámetros torácicos y disminuye la compliance pulmonar. Puede originar un cuadro similar al taponamiento cardiaco. La presión intrapleural aumentada puede incrementar la presión intrapericárdica e interferir el llenado de las cámaras cardiacas, disminuyendo el gasto cardíaco. También puede invertir el diafragma, induciendo un movimiento paradójico que disminuye la ventilación alveolar.

Estas alteraciones mejoran al drenar el DP, por lo que creemos que el DP debe ser drenado siempre que lo observemos, especialmente en presencia de insuficiencia respiratoria.
\end{abstract}

PALABRAS CLAVE: Derrame pleural. Fisiopatología. Tratamiento.

\begin{abstract}
Pleural effusion (PE) can change the equilibrium between volume of thoracic cavity and volume of intrathoracic structures, and it can disturb the function of respiratory system, heart, and diaphragm. PE alters scanty the pulmonary gas exchange, but it provokes restrictive changes in pulmonary function proportionally to fluid volume, increase thoracic diameters, and decrease lung compliance. PE can originate a syndrome similar to cardiac tamponade. Elevate intrapleural pressure increase intrapericardic pressure and this disturb the cardiac chambers filling, specially right chambers, and decrease cardiac output. Large PE can invert the diaphragm inducing paradoxical movement that origin a reduction of alveolar ventilation.

All these alterations improve with drainage of the PE. We think that $P E$ must always be drained, specially if respiratory failure is present.
\end{abstract}

KEY WORDS: Pleural effusion. Pathophysiology. Treatment.

Montero Ruiz E, Hernández Ahijado C, García Sánchez F, López Álvarez J. Consecuencias del derrame pleural. An Med Interna (Madrid) 2006; 23: 389-392.

\section{INTRODUCCIÓN}

La cavidad torácica dispone de una cierta capacidad de adaptación frente a un posible aumento del volumen de las estructuras intratorácicas. No tiene la rigidez del cráneo, que conlleva la grave consecuencia del síndrome de hipertensión intracraneal ante cualquier proceso que incremente el volumen de las estructuras intracraneales, pero tampoco es capaz de soportar la gran cantidad de líquido o los enormes tumores que admite el abdomen sin alterar la función de los órganos abdominales. Una característica propia de la cavidad torácica es que algunas de sus estructuras trabajan con presiones cercanas a cero, o incluso negativas en el caso del espacio pleural. Cualquier proceso que modifique el equilibrio entre el volumen de la cavidad torácica y el de los órganos que contiene, y que supere la capacidad de adaptación de la misma, puede provocar alteraciones en la función de los órganos torácicos.

El derrame pleural (DP) es una entidad muy frecuente en la práctica clínica. En nuestro servicio alrededor del 10\% de los enfermos ingresados lo presentan (datos no publicados), cifra que coincide con la comunicada por otros autores (1). Sin embargo, tenemos un conocimiento escaso y parcial de las consecuencias fisiopatológicas que origina en los órganos torácicos. En general, se han estudiado derrames grandes y se tiende a extrapolar los resultados a los pequeños, pero los verdaderos efectos de los DP pequeños no se conocen. Los estudios experimentales han sido realizados en animales, en la mayoría de los casos derrames unilaterales, con diferencias anatómicas y funcionales de sus tórax que pueden hacer difícil extrapolar los resultados al ser humano. Los

Trabajo aceptado: 19 de abril de 2006

Correspondencia: Eduardo Montero Ruiz. Servicio de Medicina Interna. Hospital Universitario Príncipe de Asturias. Ctra. Alcalá-Meco, s/n. 28805 Alcalá de Henares. Madrid. Fax: 918 801 825. e-mail: emonteror@wanadooadsl.net 
trabajos llevados a cabo en humanos se limitan a analizar los efectos que produce la toracocentesis evacuadora, lo cual puede dar una imagen sesgada de la fisiopatología del DP. Además, en estos últimos existe la dificultad añadida de que es muy difícil separar los efectos que produce el propio derrame de los que originan las otras patologías, pulmonares o no, presentes en el enfermo.

El DP puede provocar alteraciones en el funcionamiento del aparato respiratorio, corazón y diafragma.

\section{APARATO RESPIRATORIO}

La presencia de DP origina un aumento del espacio pleural que es compensado parcialmente por la reducción del tamaño del pulmón y la expansión de la pared torácica del mismo hemitórax (2). También puede jugar un papel en estos mecanismos compensadores el desplazamiento del mediastino hacia el hemitórax contralateral provocado por el derrame.

El DP altera el intercambio pulmonar de gases, en general en escasa cuantía, por mecanismos no bien comprendidos. La hipoxemia que origina no parece estar relacionada con cambios hemodinámicos, sino con alteraciones en el shunt intrapulmonar inducidas por la compresión del pulmón provocada por el derrame $(3,4)$. Por el contrario, al extraer el líquido pleural disminuye la compresión del pulmón y el shunt se reduce (5). En este sentido se ha observado que la evacuación del derrame mejora modestamente la perfusión de zonas mal irrigadas pero, dada la escasa influencia que tienen el volumen de líquido extraído y la velocidad del drenaje, probablemente lo que sucede es una lenta reexpansión pulmonar (3) y la consecuente lenta mejoría del shunt. También es posible que, en derrames de pequeña o moderada cantidad, el pulmón pueda estar desplazado o flotando en el líquido pleural en vez de comprimido por el mismo (6).

Si bien el tamaño del DP tiene poco efecto sobre el intercambio pulmonar de gases (3), su tiempo de evolución puede ser un factor importante desde un punto de vista fisiopatológico. En pulmones comprimidos se ha observado un aumento de la permeabilidad capilar y una alteración de la síntesis de surfactante que se incrementan con el paso del tiempo (5).

El drenaje del líquido pleural, aunque generalmente mejora el intercambio pulmonar de gases y suele reducir el gradiente alvéolo-arterial (5), puede elevar, disminuir o no afectar la $\mathrm{PaO}_{2}$ (3). Esta variabilidad sobre la $\mathrm{PaO}_{2}$ se ha intentado justificar por la presencia asociada de enfermedad pulmonar, rapidez en la formación del derrame y su naturaleza, volumen de líquido extraído o la velocidad del drenaje (3). Una posible explicación al deterioro de $1 \mathrm{a} \mathrm{PaO}_{2}$ observado en algunos casos tras la evacuación del líquido pleural puede ser la aparición de edema pulmonar ex vacuo (7). En algunas ocasiones un DP de moderada cuantía es capaz de agravar de forma importante una hipoxemia existente, en especial en presencia de insuficiencia respiratoria (4). En estos casos la extracción del líquido puede mejorar notablemente la oxigenación del enfermo.

$\mathrm{La} \mathrm{PaCO}_{2}$ se altera muy poco. Solo se eleva en presencia de DP masivo, probablemente por una disminución de la ventilación inducida por la reducción progresiva de la compliance pulmonar (4).
El DP produce alteraciones restrictivas en la función pulmonar que son proporcionales al volumen de líquido presente (8). Reduce el volumen expiratorio forzado en un segundo (FEV1), capacidad vital (VC), capacidad funcional residual (FRC), volumen residual (RV), capacidad pulmonar total (TLC) y, en escasa cuantía, la capacidad de difusión de CO (DLCO) (2). También aumenta todos los diámetros del tórax, a diferencia del neumotórax, y disminuye la compliance pulmonar (9). Existe una relación significativa entre el tamaño del derrame y el defecto ventilatorio restrictivo, ya que derrames importantes producen marcados descensos de la VC y TLC $(2,10)$. La toracocentesis evacuadora mejora significativamente (entre el 5,5 y el $18 \%$ ) el FEV1, VC, RV, TLC y DLCO. La mejora de estos parámetros está, en general, en relación bastante lineal respecto al volumen drenado (11).

El incremento de la cantidad de líquido en el espacio pleural aumenta la presión (P) intrapleural. Dicho aumento de $\mathrm{P}$ afecta a la pared torácica y al pulmón, en especial en las zonas inferiores de ambos hemitórax (12). La mejoría observada en la VC y TLC tras el drenaje del líquido pleural guarda una mayor relación con la $\mathrm{P}$ intrapleural que con el volumen de líquido extraído (13), ya que el incremento de los volúmenes pulmonares que se observa tras la toracocentesis es claramente menor que el volumen de líquido extraído (10). La toracocentesis disminuye de forma muy marcada la $\mathrm{P}$ negativa intrapleural inspiratoria (10), aunque existe mucha variación en el cambio de la $\mathrm{P}$ intrapleural obtenido después del drenaje probablemente por las diferencias individuales en la compliance del pulmón y de la pared torácica (14).

La extracción del líquido pleural produce una clara e inmediata disminución de la disnea $(5,10)$, la cual no se justifica por el modesto incremento de los volúmenes pulmonares ni por la variación obtenida en el intercambio de gases. La reducción de la disnea puede ser explicada por la mejoría de la mecánica de los músculos respiratorios que se produce al disminuir el volumen de la caja torácica al extraer el líquido pleural. Este efecto consigue que la musculatura respiratoria opere en una zona más favorable de su curva longitud-tensión (10), también es posible que disminuya la estimulación de los mecanorreceptores del pulmón y/o de la pared torácica (5), disminuyendo en ambos casos la disnea.

\section{CORAZÓN}

Se ha demostrado experimentalmente que el DP puede originar un cuadro clínico similar al taponamiento cardiaco (15), que algunos autores denominan "taponamiento torácico" (4). A pesar de esta evidencia, en la práctica clínica rara vez se toma en consideración esta posibilidad, con muy pocos casos publicados y escasa información al respecto. Sin embargo esta situación puede ser muy frecuente. En un estudio efectuado en 116 pacientes no seleccionados con DP de cualquier localización, el $18 \%$ presentaron signos ecocardiográficos de, al menos, colapso de la aurícula derecha y, también, incremento de $\mathrm{P}$ en la arteria pulmonar (16). Asimismo se han descrito casos de colapso del ventrículo izquierdo, con importante repercusión hemodinámica, secundario a DP loculado en el hemitórax izquierdo (17). 
El aumento de la presión intrapleural originado por el derrame puede ser transmitido al espacio pericárdico e incrementar la $\mathrm{P}$ de éste, lo que, a su vez, puede interferir en el llenado de las cámaras cardiacas, en especial de las derechas. Este fenómeno es capaz de originar una disminución del gasto cardiaco, exagerando los efectos de las alteraciones en la relación ventilación/perfusión que causa el DP y sus consecuencias sobre la $\mathrm{PaO}_{2}$ (4). Diversos estudios han demostrado hallazgos ecocardiográficos y hemodinámicos de taponamiento cardiaco en pacientes con DP derecho y/o izquierdo de diversas etiologías y sin derrame pericárdico significativo (17-19), hallazgos que desaparecen al drenar el derrame, a veces con extracciones de sólo $500 \mathrm{ml}$ de líquido pleural (19).

Las presiones intrapleural e intrapericárdica se elevan uniformemente durante la infusión experimental de líquido en el espacio pleural (15). El colapso diastólico del ventrículo derecho aparece, tanto en el derrame pleural como en el pericárdico, con $\mathrm{P}$ intrapericárdicas similares (15). Sin embargo, por razones no bien comprendidas, a igual $\mathrm{P}$ intrapericárdica se afecta menos el pulso venoso y se tolera mejor el derrame pleural que el pericárdico (15). El taponamiento cardiaco puede aparecer con una $\mathrm{P}$ intrapleural de 4 cm H2O, valor observado con frecuencia en la clínica (13). En estas circunstancias la toracocentesis evacuadora puede ser muy eficaz al mejorar el gasto cardíaco y el grado de oxigenación (10).

La presencia de DP, con el incremento correspondiente de la $\mathrm{P}$ intrapleural, aumenta significativamente las presiones de llenado de ambos ventrículos y la resistencia vascular pulmonar (4). Asimismo, se ha comprobado que la presión venosa central es directamente proporcional al tamaño del derrame y que, por el contrario, la $\mathrm{P}$ arterial no se ve afectada, salvo en casos muy graves de DP (4).

$\mathrm{El}$ aumento de la $\mathrm{P}$ de llenado ventricular posiblemente refleja la disminución de la compliance ventricular asociada al fenómeno progresivo de "taponamiento torácico" (4). Por otro lado, el incremento de la $\mathrm{P}$ pulmonar por mayor resistencia vascular probablemente está relacionado con la compresión física del parénquima pulmonar causada por el DP (4).

Todas las alteraciones cardiovasculares comentadas anteriormente son directamente proporcionales al tamaño del DP y son reversibles (4), lo cual tiene importantes implicaciones terapéuticas.

\section{DIAFRAGMA}

Los derrames pleurales grandes son capaces de alterar directamente la función y la anatomía del diafragma, llegando, incluso, a invertirlo. La inversión de un hemidiafragma puede estar provocada por un neumotórax a tensión, tumor del propio diafragma, masas intratorácicas o derrame pericárdico, pero la causa más habitual es el DP grande de cualquier etiología (20-23). Es menos frecuente en el lado derecho por el efecto protector del hígado (24), aunque también se han descrito casos de inversión del hemidiafragma derecho (25). Puede originar disnea, generalmente intensa, una masa abdominal palpable, dolor, distensión abdominal y disfagia si es en el lado izquierdo. Estos síntomas y signos clínicos desaparecen al drenar el líquido pleural $(20,21,26)$.
La inversión del diafragma induce un movimiento paradójico del hemidiafragma afectado (durante la inspiración se eleva en lugar de bajar) que origina una reducción de la VC y de la ventilación alveolar (20). También provoca que el aire de ese pulmón salga durante la inspiración, en vez de entrar, y vaya al pulmón contralateral, con un movimiento pendular que contribuye a la disnea (20). En estas condiciones la extracción de incluso pequeñas cantidades de líquido pleural disminuye significativamente la $\mathrm{P}$ sobre el hemidiafragma y provoca la práctica desaparición de la ventilación pendular (27). Asimismo, el drenaje mejora la VC, FEV1, $\mathrm{PaO}_{2}$ y el gradiente alvéolo-arterial, sin observarse cambios en la $\mathrm{PaCO}_{2}$ (27).

El método de elección para el diagnóstico de la inversión del diafragma, en especial la causada por un DP, es la ecografía. Puede ser difícil de diagnosticar, sobre todo si no se piensa en esta posibilidad. Algunos enfermos presentan movimiento paradójico del hemidiafragma sin clara inversión del mismo (28), comportándose como una "inversión funcional". En ocasiones el movimiento paradójico puede ser observado sólo tras algunos minutos en posición erecta y no en decúbito supino, postura habitual para la técnica de la ecografía. Este fenómeno probablemente es una consecuencia del efecto de la gravedad sobre el líquido pleural y su incidencia sobre el diafragma (28). Estas circunstancias pueden hacer que estos enfermos no sean diagnosticados correctamente.

\section{CONCLUSIONES}

En el momento actual conocemos poco las consecuencias que conlleva la presencia de un DP. En el aparato respiratorio modifica poco el intercambio de gases, pero produce alteraciones ventilatorias restrictivas que pueden ser importantes y que guardan relación directa con el tamaño del derrame. Sobre el corazón puede causar una situación similar al taponamiento cardiaco, a veces con graves repercusiones hemodinámicas. En el diafragma el DP es capaz de provocar una inversión del mismo, originando un movimiento pendular del aire entre ambos pulmones con importantes repercusiones funcionales respiratorias. El síntoma fundamental que provoca el DP es la disnea, inducida por mecanismos no bien comprendidos y que probablemente estén relacionados en mayor o menor medida con los tres órganos afectados. Una característica común, tanto de la disnea como de todas las alteraciones fisiopatológicas comentadas anteriormente, es que mejoran, en general de forma muy marcada, al drenar el líquido pleural.

Nuestra opinión, fundamentados en los conocimientos actuales sobre su fisiopatología, es que el DP debe ser drenado siempre que lo observemos, en especial si el enfermo presenta insuficiencia respiratoria, máxime teniendo en cuenta que la gran mayoría de los pacientes tienen patologías asociadas que los deterioran aún más. La única excepción es el hidrotórax secundario a ascitis, la cual debe ser corregida antes de drenar el DP. La técnica del drenaje, realizada con un mínimo de precauciones, es rápida, prácticamente inocua y si la hacemos correctamente no aparecerán las complicaciones más importantes, el neumotórax yatrógeno y el edema pulmonar ex vacuo. 


\section{Bibliografía}

1. Martínez-Berganza A, Cía P. Epidemiología de las enfermedades de la pleura. A propósito de 562 casos. Med Clin (Barc) 1988; 90: 311-5.

2. Gilmartin JJ, Wright AJ, Gibson GJ. Effects of pneumothorax or pleural effusion on pulmonary function. Thorax 1985; 40: 60-5.

3. Agustí AGN, Cardús J, Roca J, Grau JM, Xaubet A, Rodríguez-Roisin R. Ventilation-perfusion mismatch in patients with pleural effusion. Effects of thoracentesis. Am J Respir Crit Care Med 1997; 156: 1205-9.

4. Nishida O, Arellano R, Cheng DCH, DeMajo W, Kavanagh BP. Gas exchange and hemodynamics in experimental pleural effusion. Crit Care Med 1999; 27: 583-7.

5. Perpiñá M, Benlloch E, Marco V, Abad F, Nauffal D. Effect of thoracentesis on pulmonary gas exchange. Thorax 1983; 38: 747-50.

6. Anthonisen NR, Martin RR. Regional lung function in pleural effusion. Am Rev Respir Dis 1977; 116: 201-7.

7. Doerschuk CM, Allard MF, Oyarzun MJ. Evaluation of reexpansion pulmonary edema following unilateral pneumothorax in rabbits and the effect of superoxide dismutase. Exp Lung Res 1990; 16: 355-67.

8. Yoo OH, Ting EY. The effects of pleural effusion on pulmonary function. Am Rev Respir Dis 1964; 89: 55-63.

9. Sousa AS, Moll RJ, Pontes CF, Saldiva PHN, Zin WA. Mechanical and morphometrical changes in progressive bilateral pneumothorax and pleural effusion in normal rats. Eur Respir J 1995; 8: 99-104.

10. Estenne M, Yernault JC, De Troyer A. Mechanism of relief of dyspnea after thoracocentesis in patients with large pleural effusions. Am J Med 1983; 74: 813-9.

11. Zerahn B, Jensen BV, Olsen F, Petersen JR, Kanstrup IL. The effect of thoracentesis on lung function and transthoracic electrical bioimpedance. Respir Med 1999; 93: 196-201.

12. Krell WS, Rodarte JR. Effects of acute pleural effusion on respiratory system mechanics in dogs. J Appl Physiol 1985; 59: 1458-63.

13. Light RW, Stansbury DW, Brown SE. The relationship between pleural pressures and changes in pulmonary function after therapeutic thoracentesis. Am Rev Respir Dis 1986; 133: 658-61.

14. Light RW, Jenkinson SG, Minh VD, George RB. Observations on pleural fluid pressures as fluid is withdrawn during thoracentesis. Am Rev Respir Dis 1980; 121: 799-804.

15. Vaska K, Wann LS, Sagar K, Klopfenstein HS. Pleural effusion as a cause of right ventricular diastolic collapse. Circulation 1992; 86: 60917.

16. Sadaniantz A, Anastacio R, Verma V, Aprahamian N. The incidente of diastolic right atrial collapse in patients with pleural effusion in the absence of pericardial effusion. Echocardiography 2003; 20: 211-5.

17. Kisanuki A, Shono H, Kiyonaga K, Kawataki M, Otsugi Y, Minagoe S, et al. Two-dimensional echocardiographic demonstration of left ventricular diastolic collapse due to compression by pleural effusion. Am Heart J 1991; 122: 1173-5.

18. Kaplan LM, Epstein SK, Schwartz SL, Cao QL, Pandian NG. Clinical, echocardiographic, and hemodynamic evidence of cardiac tamponade caused by large pleural effusions. Am J Respir Crit Care Med 1995; 151: 904-8.

19. Alam HB, Levitt A, Molyneaux R, Davidson P, Sample GA. Can pleural effusions cause cardiac tamponade? Chest 1999; 116: 1820-2.

20. Mulvey RB. The effect of pleural fluid on the diaphragm. Radiology 1965; 84: 1080-6.

21. Swingle JD, Logan R, Juhl JH. Inversion of the left hemidiaphragm JAMA 1969; 208: 863-4.

22. Rogers CI, Meredith HC. Osler revisited: an unusual cause of inversion of the diaphragm. Radiology 1977; 125: 596.

23. Yeh HC, Halton KP, Gray CE. Anatomic variations and abnormalities in the diaphragm seen with US. Radiographics 1990; 10: 1019-30.

24. Katzen BT, Choi WS, Friedman MH, Green IJ, Hindle WV, Zellis A. Pseudomass of the liver due to pleural effusion and inversion of the diaphragm. AJR 1978; 131: 1077-8.

25. Lowe SH, Cosgrove DO, Joseph AEA. Inversion of the right hemidiaphragm shown on ultrasound examination. Br J Radiol 1981; 54: 754-7.

26. Dallemand S, Twersky J, Gordon DH. Pseudomass of the left upper quadrant from inversion of the left hemidiaphragm: CT diagnosis. Gastrointest Radiol 1982; 7: 57-9.

27. Wang JS, Tseng H. Changes in pulmonary mechanics and gas exchange after thoracentesis on patients with inversion of a hemidiaphragm secondary to large pleural effusion. Chest 1995; 107: 1610-4.

28. Cooper JCE, Elliott ST. Pleural effusions, diaphragm inversion, and paradox: new observations using sonography. AJR 1995; 164: 510. 